\title{
Performance-Based Risk-Sharing Arrangements for Pharmaceutical Products in the United States: A Systematic Review
}

\author{
Justin S. Yu, PharmD, MS; Lauren Chin, PharmD; Jennifer Oh, BS; and Jorge Farias, PharmD, MS
}

\begin{abstract}
BACKGROUND: Value for money is a growing necessity in today's U.S. health care system in which drug spending is expected to increase by an average rate of $6.7 \%$ yearly through 2025 . In response to uncertainty about real-world clinical and economic outcomes for many drugs, health insurers and pharmacy benefit managers (PBMs) have implemented various contracts and arrangements with drug manufacturers that can collectively be described as performance-based risk-sharing arrangements (PBRSAs). Little is known about U.S.-specific PBRSAs for drugs.
\end{abstract}

OBJECTIVES: To conduct a systematic review of U.S.-specific PBRSAs for drugs to describe (a) trends over time and (b) key aspects including outcome measures and terms of arrangements between stakeholders.

METHODS: A systematic review was conducted in MEDLINE (January 1, 1946-April 1, 2017), Embase (January 1, 1988-April 1, 2017), and the grey literature (up to April 1, 2017) to identify publicly disclosed PBRSAs. Articles and conference abstracts were included if they were published in English and described a U.S.-specific PBRSA for a drug. Articles and conference abstracts were excluded if they only described a PBRSA similar to a money-back guarantee to patients. They were also excluded if they only described a PBRSA between a PBM and a health insurer in which the latter would receive a discount for patients nonadherent to a drug. Results were summarized as counts and percentages.

RESULTS: From the literature review, 26 publicly disclosed PBRSAs were identified. Of these, 16 (62\%) were announced or initiated from 2015 to 2017 , and 10 (38\%) were announced or initiated from 1997 to 2012. Thirteen (50\%) PBRSAs involved cardiometabolic indications; 5 (19\%) involved oncology indications; and $8(31 \%)$ involved other indications. Categorized by health insurer or PBM, 10 (38\%) PBRSAs involved large multistate insurers; $5(19 \%)$ involved the Centers for Medicare \& Medicaid Services; 7 (27\%) involved regional insurers; 3 (12\%) involved PBMs; and 1 $(4 \%)$ involved multiple unspecified insurers. Regarding the most active drug manufacturers, Amgen initiated 5 (19\%) PBRSAs and Novartis initiated 4 (15\%). Relative to the initial FDA approval of a treatment, $15(58 \%)$ PBRSAs were announced or initiated within 5 years, and $11(42 \%)$ were announced or initiated more than 5 years later. For data collection, electronic medical record (EMR) data would have been an appropriate source for $12(46 \%)$ PBRSAs; claims data would have been an appropriate source for 11 (42\%) PBRSAs; and EMR and claims data would have been appropriate sources for $2(8 \%)$ PBRSAs; no description of the outcome measures was available for 1 (4\%) PBRSA.

CONCLUSIONS: The number of publicly disclosed U.S.-specific PBRSAs for drugs has increased over the years. This review's findings confirm the interest of stakeholders in such arrangements and their confidence in the use of the selected outcome measures. Each PBRSA represents a timely collaboration among stakeholders to provide access to a drug while generating evidence to better elucidate its clinical and economic value.

J Manag Care Spec Pharm. 2017;23(10):1028-40

Copyright $\odot 2017$, Academy of Managed Care Pharmacy. All rights reserved.

\section{What is already known about this subject}

Because of uncertainty about real-world clinical and economic outcomes for many drugs, health insurers and pharmacy benefit managers have implemented various contracts and arrangements with drug manufacturers that can collectively be described as performance-based risk-sharing arrangements (PBRSAs).

A 2010 international review of PBRSAs identified 8 U.S. specific PBRSAs for the drugs oxaliplatin, irinotecan, cetuximab, bevacizumab, erythropoiesis-stimulating agents, simvastatin, sitagliptin and sitagliptin-metformin, and risedronate sodium.

\section{What this study adds}

This systematic review identified 26 U.S.-specific PBRSAs for drugs announced or initiated from 1997 to 2017

This review summarized information related to corresponding drugs, factors surrounding the PBRSAs, stakeholders involved in the PBRSAs, and outcome measures used in the PBRSAs.

T alue in health care can be defined in its simplest form as health outcomes achieved per dollar spent on medical care. ${ }^{1}$ As an extension of this concept, value-based pricing is the price of health care services that reflects clinical and economic benefits., ${ }^{2,3}$ Although prices of drugs are determined at their launch, finite health care resources and uncertainty around real-world clinical outcomes often limit the uptake of new, innovative treatments. ${ }^{4,5}$ Pharmaceutical companies have identified these issues as key factors contributing to less-thanexpected usage and revenue for newly launched drugs. ${ }^{4,5}$ With an increase in drug spending over time, especially for specialty drugs, ${ }^{6}$ health care decision makers have placed great importance on understanding the value of drugs in order to inform purchasing decisions, manage drug spending, and improve the delivery of care. ${ }^{7}$

In response to uncertainty around clinical outcomes for specific drugs, health insurers have implemented various contracts and arrangements with drug manufacturers, which can collectively be described as performance-based risksharing arrangements (PBRSAs). Such arrangements include, but are not limited to, value-based contracts, outcomes-based contracts, risk-sharing agreements, coverage with evidence development (CED), and managed entry schemes. ${ }^{8}$ Using the Performance Based Risk Sharing Database, which is 
available at the University of Washington School of Pharmacy, a 2014 review of publicly disclosed PBRSAs revealed that Italy had the most arrangements $(n=36)$, followed by the United Kingdom ( $n=29)$, Netherlands $(n=23)$, United States $(n=18)$, and Sweden ( $\mathrm{n}=17$ ) from January 1993 to June 2013. ${ }^{9}$ Among these arrangements, the most represented therapeutic areas were oncology $(n=77)$, endocrinology $(n=14)$, neurology $(n=10)$, and cardiology $(n=10)$, and the majority $(92 \%)$ were for drugs rather than devices or diagnostic tests. ${ }^{9}$

In contrast to other countries in the years leading up to 2013, PBRSAs in the United States focused primarily on CED. ${ }^{9}$ In addition, the majority of these PBRSAs focused largely on devices and surgical procedures as opposed to drugs. ${ }^{10}$ CED involves an agreement by a health insurer to provide broader coverage for a promising treatment despite evidence gaps and is contingent on the facilitation of additional collection of data. ${ }^{11}$ In recent years (2015 to present), however, U.S. interest in drug-related PBRSAs appears to have been revived, driven largely by the launch of new therapies with high price tags for potentially large patient populations (e.g., sacubitril-valsartan, evolocumab, and alirocumab) or for smaller populations but with potentially larger budget impacts (e.g., sofosbuvir and ledipasvir-sofosbuvir).

In response to these demands on the health care system, professional organizations such as the American Society of Clinical Oncology, National Comprehensive Cancer Network, and the Institute for Clinical and Economic Review have developed frameworks and reports that focus on the value of drugs while taking into account economic burden. ${ }^{12-14}$ Memorial Sloan Kettering Cancer Center also released DrugAbacus, which incorporates inputs such as dollars per year, toxicity, novelty, rarity, population burden of disease, cost of development, prognosis, and unmet need to estimate the appropriate prices of oncology drugs based on their perceived value. ${ }^{15}$ With specialty drug spending expected to account for approximately $50 \%$ of total annual pharmacy spending by $2018,{ }^{16-18}$ in combination with the lack of real-world evidence and robust comparative information of the benefits of many drugs, ${ }^{7}$ PBRSAs can serve as an option to facilitate better value for health care dollars.

Value for money is vital to a health care system that continues to trend upwards in terms of overall drug spending, despite finite health care resources-prescription drug spending is expected to increase by an average rate of $6.7 \%$ yearly through 2025 , exceeding that of total health care spending (i.e., 5.8\%). ${ }^{19}$ In view of the lack of a systematic review of U.S.-specific PBRSAs for drugs, the objectives of this review were to (a) provide a greater understanding of PBRSA trends over the years and (b) describe key aspects, including outcome measures and terms, of the arrangements between stakeholders

\section{Methods}

\section{Search Strategy and Selection Criteria}

A systematic review was first conducted in MEDLINE (January 1, 1946-April 1, 2017) and Embase (January 1, 1988-April 1, 2017) using the following search terms and their nonhyphenated versions (when applicable) as key words: value-based pricing, value-based contract*, value-based agreement*, performance-based agreement*, performance-based scheme*, price-volume agreement*, price-volume arrangement*, outcomes-based contract*, outcomesbased agreement*, coverage with evidence, conditional coverage, conditional reimbursement, risk-sharing agreement*, risk-sharing arrangement*, outcome guarantee*, health impact guarantee*, pay back scheme*, denial of payment, paying for outcomes, and no cure no pay. These search terms were selected based on a review of published literature and grey literature in order to maximize the sensitivity of the search strategy to identify PBRSAs. Articles and conference abstracts were evaluated to minimize any loss of relevant information.

To ensure completeness of the literature review, and using the same search terms, a search of the grey literature (up to April 1 , 2017) was performed using Google; the Centers for Medicare \& Medicaid Services website; 2015 annual reports of the top 20 pharmaceutical companies by revenue (Pfizer, Roche, Merck, Gilead Sciences, Sanofi, Johnson \& Johnson, Novartis, GlaxoSmithKline, AstraZeneca, AbbVie, Amgen, Eli Lilly, Bristol-Myers Squibb, Novo Nordisk, Bayer, Takeda, Boehringer Ingelheim, Astellas, Biogen, and Otsuka) ${ }^{20} ; 2015$ annual reports of the top 10 health insurance companies by market value (UnitedHealth Group, Cigna, Anthem, Humana, Aetna, Molina Healthcare, Centene Corporation, Magellan Health, WellCare, and Health Net) ${ }^{21}$; and 2015 annual reports of the top 3 pharmacy benefit managers (PBMs) by covered lives (Express Scripts, CVS Health, and OptumRx). ${ }^{22}$

Inclusion criteria for this systematic review were defined a priori. Articles (e.g., peer-reviewed manuscripts, press releases, and news articles) and conference abstracts were included only if they were in English and mentioned a U.S.-specific PBRSA. Annual reports before 2015 were only evaluated if a PBRSA was identified in the 2015 report (most recent year available at the time of this review). For the grey literature search, only the earliest-identified articles for PBRSAs and articles with additional information on those PBRSAs were included. Articles and conference abstracts were excluded if they only described PBRSAs that were similar to money-back guarantees to patients. They were also excluded if they only described PBRSAs between PBMs and health insurers, in which the latter would receive discounts for patient nonadherence to a drug (this type of PBRSA focuses more on the value of PBM services than on the value of a drug). Given that this systematic review focused on 


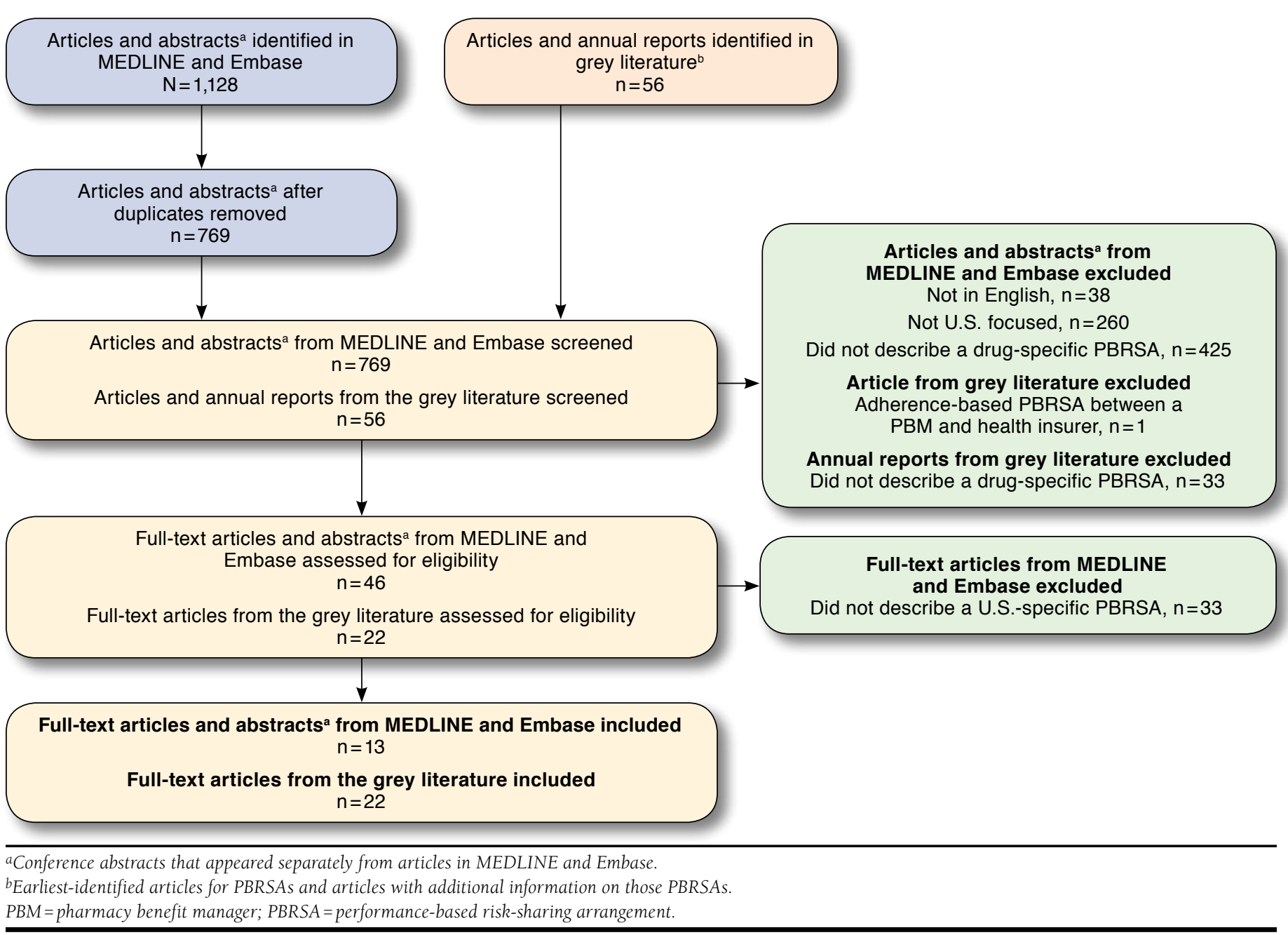

the identification of PBRSAs, the PICOS criteria (participants, interventions, comparators, outcomes, study design) were not considered applicable.

After the articles and conference abstracts were retrieved, 2 of the review authors (JY and LC) independently reviewed each article and conference abstract by first screening the title and abstract to determine eligibility for inclusion in the study results. When it was unclear if an article referred to a PBRSA, the article was scheduled for a full-text review. All disagreements were discussed until a consensus was reached. After identifying relevant articles and conference abstracts, the grey literature was reviewed to understand details on the drugs associated with the PBRSAs. The sources of information reviewed included drug prescribing information and the U.S. Food and Drug Administration (FDA) website.

\section{Data Extraction and Analyses}

One review author (JY) extracted data from each article and conference abstract that met the selection criteria, including the year the PBRSA was announced or initiated, products, disease state, drug manufacturers, health insurer or PBM, description of the PBRSA, and outcome measures of interest. Data on the drug approval year, trade name, same-class competitors and same disease-state competitors, and time from drug approval since the PBRSA was announced or initiated were also recorded based on a review of the grey literature. In addition, based on the primary uses of electronic medical record (EMR) and claims data (for clinical and billing purposes, respectively) and their widespread use, ${ }^{23}$ each PBRSA was classified according to its likelihood of using each data type to assess the outcome measures. Other data sources, however, could also be used and 


\section{TABLE 1 Summary of Literature Review}

\begin{tabular}{|c|c|c|c|c|c|c|c|c|c|c|}
\hline 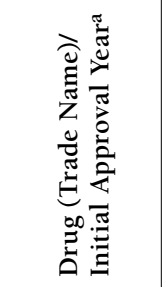 & 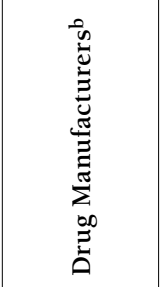 & 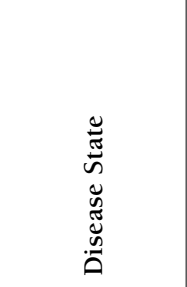 & 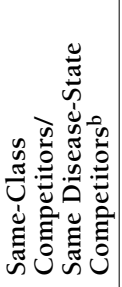 & 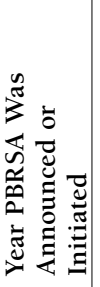 & 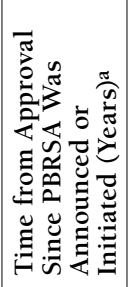 & 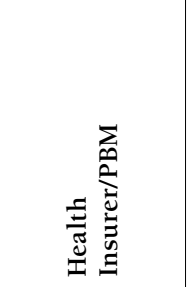 & 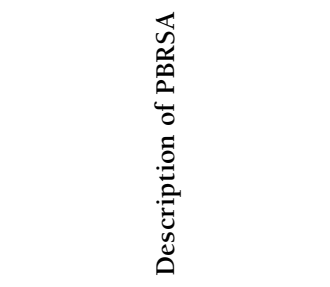 & 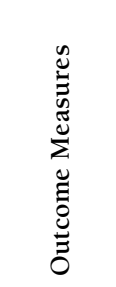 & 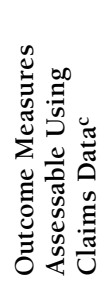 & 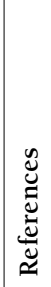 \\
\hline $\begin{array}{l}\text { Epoetin alfa } \\
\text { (Epogen, } \\
\text { Procrit) } \\
1989\end{array}$ & $\begin{array}{l}\text { Amgen, } \\
\text { Ortho } \\
\text { Biotech }\end{array}$ & Anemia & Yes/Yes & 1997 & 8 & $\begin{array}{c}\text { Health Care } \\
\text { Financing } \\
\text { Administration } \\
\text { (CMS) }\end{array}$ & $\begin{array}{l}\text { CMS will use a 3-month } \\
\text { rolling average Hct level } \\
\text { for reimbursement of } \\
\text { epoetin alfa claims in } \\
\text { hemodialysis patients } \\
\text { with Hct greater than } \\
36 \% \text { in any given month, } \\
\text { with denial of payment } \\
\text { when the 3-month rolling } \\
\text { average exceeds } 36.5 \% \text {. }\end{array}$ & Hct & No & $\begin{array}{l}10, \\
27, \\
58\end{array}$ \\
\hline $\begin{array}{l}\text { Simvastatin } \\
\text { (Zocor) } \\
1991\end{array}$ & Merck & Hyperlipidemia & Yes/Yes & 1998 & 7 & $\begin{array}{l}\text { Patients and } \\
\text { insurers }\end{array}$ & $\begin{array}{l}\text { Merck will refund patients } \\
\text { and insurers up to } 6 \\
\text { months of prescription } \\
\text { costs if simvastatin plus } \\
\text { diet does not help lower } \\
\text { LDL cholesterol to target } \\
\text { concentrations identified } \\
\text { by their doctors. }\end{array}$ & LDL & No & $\begin{array}{l}25 \\
58\end{array}$ \\
\hline $\begin{array}{l}\text { Oxaliplatin } \\
\text { (Eloxatin) } \\
2002\end{array}$ & $\begin{array}{l}\text { Sanofi- } \\
\text { Aventis }\end{array}$ & $\begin{array}{l}\text { Colorectal } \\
\text { cancer }\end{array}$ & $\begin{array}{c}\text { N/A } \\
\text { (CED)/ } \\
\text { N/A } \\
\text { (CED) }\end{array}$ & 2005 & 3 & CMS & \multirow{4}{*}{$\begin{array}{l}\text { CMS will cover oxalipla- } \\
\text { tin, irinotecan, cetuximab, } \\
\text { and bevacizumab for the } \\
\text { off-label treatment of } \\
\text { colorectal cancer in the } \\
\text { context of an approved } \\
\text { clinical trial. }\end{array}$} & Survival & No & \multirow{4}{*}{$\begin{array}{l}24 \\
52 \\
53 \\
58\end{array}$} \\
\hline $\begin{array}{l}\text { Irinotecan } \\
\text { (Camptosar) } \\
1996\end{array}$ & Pfizer & $\begin{array}{l}\text { Colorectal } \\
\text { cancer }\end{array}$ & $\begin{array}{c}\text { N/A } \\
\text { (CED)/ } \\
\text { N/A } \\
(\mathrm{CED}) \\
\end{array}$ & 2005 & 9 & CMS & & Survival & No & \\
\hline $\begin{array}{l}\text { Cetuximab } \\
\text { (Erbitux) } \\
2004\end{array}$ & $\begin{array}{c}\text { ImClone, } \\
\text { Bristol-Myers } \\
\text { Squibb }\end{array}$ & $\begin{array}{l}\text { Colorectal } \\
\text { cancer }\end{array}$ & $\begin{array}{c}\text { N/A } \\
\text { (CED)/ } \\
\text { N/A } \\
\text { (CED) }\end{array}$ & 2005 & 1 & CMS & & Survival & No & \\
\hline $\begin{array}{l}\text { Bevacizumab } \\
\text { (Avastin) } \\
2004\end{array}$ & Genentech & $\begin{array}{l}\text { Colorectal } \\
\text { cancer }\end{array}$ & $\begin{array}{c}\text { N/A } \\
\text { (CED)/ } \\
\text { N/A } \\
(\mathrm{CED}) \\
\end{array}$ & 2005 & 1 & CMS & & Survival & No & \\
\hline $\begin{array}{l}\text { Sitagliptin } \\
\text { (Januvia) } \\
2006\end{array}$ & \multirow[b]{2}{*}{ Merck } & \multirow[b]{2}{*}{$\begin{array}{l}\text { Type } 2 \\
\text { diabetes } \\
\text { mellitus }\end{array}$} & Yes/Yes & & 3 & & \multirow{2}{*}{$\begin{array}{l}\text { Merck will tie what Cigna } \\
\text { pays for sitagliptin and } \\
\text { sitagliptin-metformin to } \\
\text { how well type } 2 \text { diabetes } \\
\text { patients are able to control } \\
\text { blood sugar. }\end{array}$} & \multirow[b]{2}{*}{$\begin{array}{l}\text { Blood } \\
\text { sugar }\end{array}$} & \multirow[b]{2}{*}{ No } & \\
\hline $\begin{array}{l}\text { Sitagliptin } \\
\text { and } \\
\text { metformin } \\
\text { (Janumet) } \\
2007\end{array}$ & & & Yes/Yes & 2009 & 2 & Cigna & & & & $\begin{array}{l}34, \\
58\end{array}$ \\
\hline
\end{tabular}

continued on next page

varied based on each PBRSA. After the initial data extraction, the other review authors independently reviewed the data table to ensure accuracy of the findings. Note that the risk of bias was not assessed for each included article, since the objective of this systematic review was to identify PBRSAs rather than to assess the validity of study outcomes. Results were summarized as counts and percentages.

\section{Results}

We retrieved and screened 472 articles and 297 abstracts (769 records total) from MEDLINE and Embase. After applying the inclusion and exclusion criteria, 11 peer-reviewed $\operatorname{articles}^{8-10,24-31}$ and 2 conference abstracts ${ }^{32,33}$ were eligible for inclusion. The grey literature search revealed 22 articles that were eligible for inclusion (Figure 1). ${ }^{34-55}$ In the event that 


\section{TABLE 1 Summary of Literature Review (continued)}

\begin{tabular}{|c|c|c|c|c|c|c|c|c|c|c|}
\hline 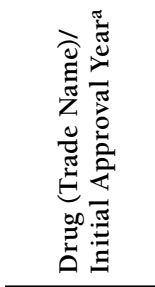 & 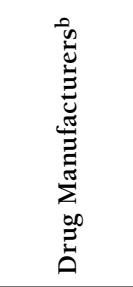 & 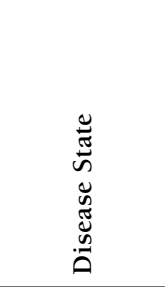 & 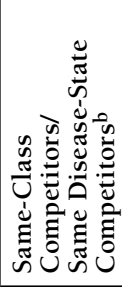 & 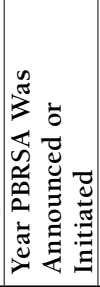 & 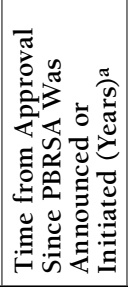 & 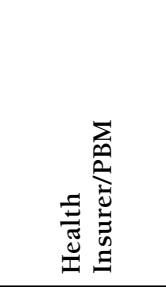 & 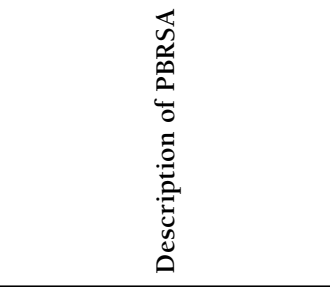 & 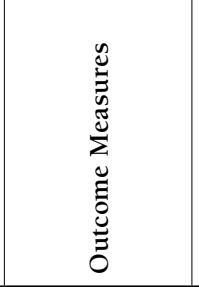 & 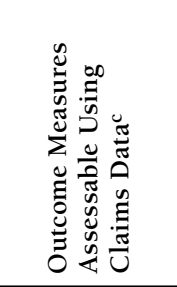 & 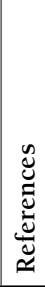 \\
\hline $\begin{array}{l}\text { Risedronate } \\
\text { (Actonel) } \\
1998\end{array}$ & $\begin{array}{l}\text { Procter \& } \\
\text { Gamble, } \\
\text { Sanofi- } \\
\text { Aventis }\end{array}$ & Osteoporosis & Yes/Yes & 2009 & 11 & $\begin{array}{c}\text { Health } \\
\text { Alliance }\end{array}$ & $\begin{array}{l}\text { Procter \& Gamble and } \\
\text { Sanofi will reimburse } \\
\text { Health Alliance for } \\
\text { the costs of treating } \\
\text { nonspinal fractures } \\
\text { suffered by patients taking } \\
\text { risedronate. }\end{array}$ & $\begin{array}{l}\text { Nonspinal } \\
\text { fracture }\end{array}$ & Yes & $\begin{array}{l}35, \\
58\end{array}$ \\
\hline $\begin{array}{l}\text { Interferon } \\
\text { beta-la } \\
\text { (Rebif) } \\
1996\end{array}$ & $\begin{array}{l}\text { EMD } \\
\text { Serono }\end{array}$ & $\begin{array}{l}\text { Multiple } \\
\text { sclerosis }\end{array}$ & Yes/Yes & 2011 & 15 & Cigna & $\begin{array}{l}\text { Cigna will track the per- } \\
\text { centage of hospitalization } \\
\text { and emergency room } \\
\text { visits avoided by people } \\
\text { using interferon beta-la by } \\
\text { tracking medical claims } \\
\text { in order to determine } \\
\text { whether a relapse was } \\
\text { the cause. The company } \\
\text { will use } 2010 \text { as a base- } \\
\text { line, looking at data from } \\
\text { medical, pharmacy, and } \\
\text { lab claims, and measure } \\
\text { members' MPR. This } \\
\text { represents a multitiered } \\
\text { contract where discounts } \\
\text { will be based on ranges for } \\
\text { medication adherence, and } \\
\text { then on customers with } \\
\text { multiple sclerosis being } \\
\text { relapse-free. }\end{array}$ & $\begin{array}{l}\text { Hospitalization, } \\
\text { emergency } \\
\text { room visit, } \\
\text { adherence }\end{array}$ & Yes & $\begin{array}{l}36, \\
37, \\
58\end{array}$ \\
\hline $\begin{array}{l}\text { Interferon } \\
\text { beta-la } \\
\text { (Rebif) } \\
1996\end{array}$ & $\begin{array}{l}\text { EMD } \\
\text { Serono }\end{array}$ & $\begin{array}{l}\text { Multiple } \\
\text { sclerosis }\end{array}$ & Yes/Yes & 2012 & 16 & $\begin{array}{c}\text { Prime } \\
\text { Therapeutics } \\
\text { (PBM) }\end{array}$ & $\begin{array}{l}\text { EMD Serono will } \\
\text { pay rebates to Prime } \\
\text { Therapeutics for use } \\
\text { of interferon beta-la if } \\
\text { patients who are on the } \\
\text { drug have a higher overall } \\
\text { total cost for their plans } \\
\text { than patients on a differ- } \\
\text { ent multiple sclerosis drug, } \\
\text { or if medication adher- } \\
\text { ence rate remains above } \\
\text { a specified level. Prime } \\
\text { Therapeutics will evaluate } \\
\text { data pertaining to health } \\
\text { outcomes and medication } \\
\text { adherence of patients a } \\
\text { number of times through- } \\
\text { out the contract in order } \\
\text { to determine total cost of } \\
\text { care, including medical } \\
\text { and pharmacy costs. }\end{array}$ & Cost, adherence & Yes & $\begin{array}{l}38, \\
58\end{array}$ \\
\hline $\begin{array}{l}\text { Fingolimod } \\
\text { (Gilenya) } \\
2010\end{array}$ & Novartis & $\begin{array}{l}\text { Multiple } \\
\text { sclerosis }\end{array}$ & No/Yes & 2015 & 5 & Humana & Not available & Not available & Not available & $\begin{array}{l}39, \\
58\end{array}$ \\
\hline
\end{tabular}




\section{TABLE 1 Summary of Literature Review (continued)}

\begin{tabular}{|c|c|c|c|c|c|c|c|c|c|c|}
\hline 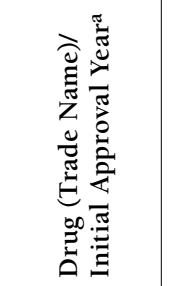 & 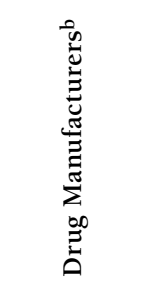 & 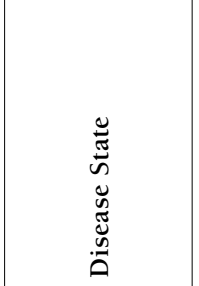 & 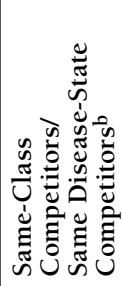 & 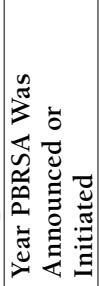 & 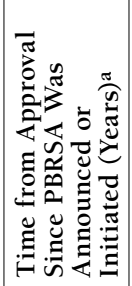 & & 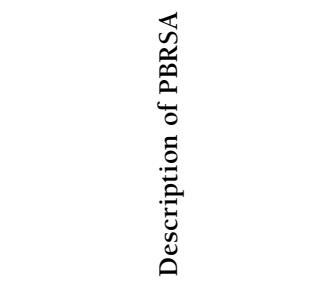 & 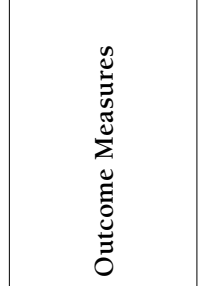 & 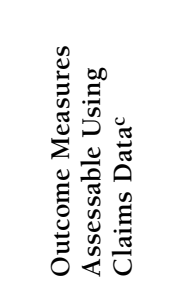 & 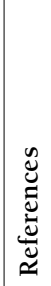 \\
\hline $\begin{array}{l}\text { Ledipasvir } \\
\text { and } \\
\text { sofosbuvir } \\
\text { (Harvoni) } \\
2014\end{array}$ & Gilead & Hepatitis C & Yes/Yes & 2015 & 1 & Cigna & $\begin{array}{l}\text { An innovative outcomes } \\
\text { incentive alignment } \\
\text { will be developed based } \\
\text { on actual SVR results } \\
\text { across Cigna's customer } \\
\text { population. }\end{array}$ & SVR & No & $\begin{array}{l}40 \\
58\end{array}$ \\
\hline $\begin{array}{l}\text { Evolocumab } \\
\text { (Repatha) } \\
2015\end{array}$ & Amgen & Hyperlipidemia & Yes/Yes & 2015 & 0 & $\begin{array}{l}\text { CVS Health } \\
\text { (PBM) }\end{array}$ & $\begin{array}{l}\text { Amgen will link the net } \\
\text { price of evolocumab to } \\
\text { expected LDL cholesterol } \\
\text { reductions and anticipated } \\
\text { appropriate patient use. }\end{array}$ & LDL & No & $\begin{array}{l}50 \\
58\end{array}$ \\
\hline $\begin{array}{l}\text { Evolocumab } \\
\text { (Repatha) } \\
2015\end{array}$ & Amgen & Hyperlipidemia & Yes/Yes & 2016 & 1 & $\begin{array}{l}\text { Harvard } \\
\text { Pilgrim }\end{array}$ & $\begin{array}{l}\text { Amgen will provide } \\
\text { Harvard Pilgrim with an } \\
\text { enhanced discount if the } \\
\text { reduction in LDL levels } \\
\text { for Harvard Pilgrim mem- } \\
\text { bers is less than what was } \\
\text { observed during clinical } \\
\text { trials. In addition to the } \\
\text { outcomes guarantee, the } \\
\text { agreement will provide } \\
\text { for additional discounts } \\
\text { if utilization of the drug } \\
\text { exceeds certain levels. The } \\
\text { contract also contains an } \\
\text { adherence provision that } \\
\text { conditions discounts on } \\
\text { whether patients reliably } \\
\text { take the drug. }\end{array}$ & LDL, adherence & $\begin{array}{l}\text { No (LDL), Yes } \\
\text { (adherence) }\end{array}$ & $\begin{array}{l}26, \\
51, \\
58\end{array}$ \\
\hline $\begin{array}{l}\text { Alirocumab } \\
\text { (Praluent) } \\
2015\end{array}$ & $\begin{array}{l}\text { Sanofi, } \\
\text { Regeneron }\end{array}$ & Hyperlipidemia & Yes/Yes & 2016 & 1 & Cigna & $\begin{array}{l}\text { Sanofi/Regeneron will } \\
\text { further discount the cost } \\
\text { of alirocumab if Cigna's } \\
\text { customers are not able to } \\
\text { reduce LDL levels at least } \\
\text { as well as what was expe- } \\
\text { rienced in clinical trials. }\end{array}$ & LDL & No & $\begin{array}{l}41, \\
58\end{array}$ \\
\hline $\begin{array}{l}\text { Evolocumab } \\
\text { (Repatha) } \\
2015\end{array}$ & Amgen & Hyperlipidemia & Yes/Yes & 2016 & 1 & Cigna & $\begin{array}{l}\text { Amgen will further } \\
\text { discount the cost of evo- } \\
\text { locumab if Cigna's custom- } \\
\text { ers are not able to reduce } \\
\text { LDL levels at least as well } \\
\text { as what was experienced } \\
\text { in clinical trials. }\end{array}$ & LDL & No & $\begin{array}{l}41, \\
58\end{array}$ \\
\hline $\begin{array}{l}\text { Sacubitril } \\
\text { and valsartan } \\
\text { (Entresto) } \\
2015\end{array}$ & Novartis & $\begin{array}{l}\text { Chronic heart } \\
\text { failure }\end{array}$ & No/Yes & 2016 & 1 & Cigna & $\begin{array}{l}\text { The arrangement ties } \\
\text { financial terms to how } \\
\text { well sacubitril-valsartan } \\
\text { improves the relative } \\
\text { health of Cigna's custom- } \\
\text { ers. The primary outcome } \\
\text { measure is the reduction } \\
\text { in proportion of customers } \\
\text { with heart failure hospital- } \\
\text { izations. }\end{array}$ & Hospitalization & Yes & $\begin{array}{l}42, \\
43, \\
58\end{array}$ \\
\hline
\end{tabular}




\section{TABLE 1 Summary of Literature Review (continued)}

\begin{tabular}{|c|c|c|c|c|c|c|c|c|c|c|}
\hline 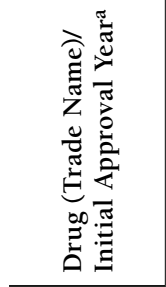 & 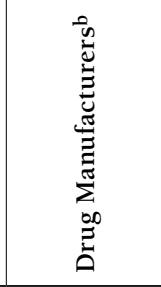 & 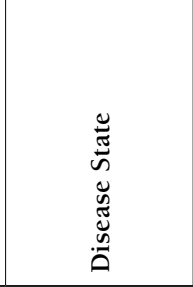 & 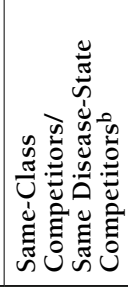 & 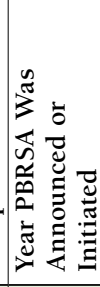 & 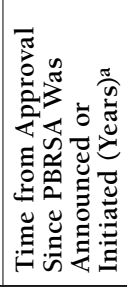 & 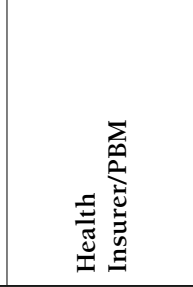 & 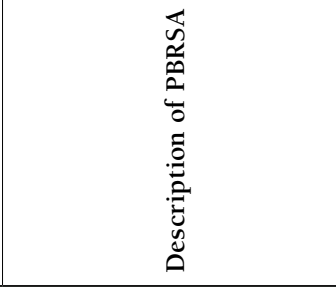 & 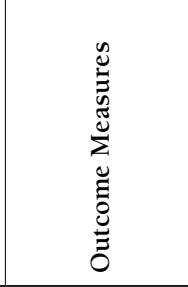 & 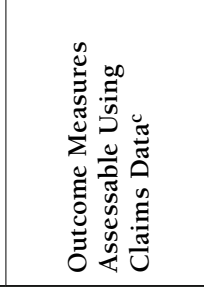 & $\frac{\mathscr{U}}{\mathscr{U}}$ \\
\hline $\begin{array}{l}\text { Sacubitril } \\
\text { and valsartan } \\
\text { (Entresto) } \\
2015\end{array}$ & Novartis & $\begin{array}{l}\text { Chronic heart } \\
\text { failure }\end{array}$ & No/Yes & 2016 & 1 & Aetna & $\begin{array}{l}\text { The arrangement is based } \\
\text { on sacubitril-valsartan } \\
\text { replicating results } \\
\text { achieved in clinical } \\
\text { trials (i.e., reduction } \\
\text { in hospitalizations and } \\
\text { cardiovascular deaths } \\
\text { related to heart failure). }\end{array}$ & $\begin{array}{c}\text { Hospitalization, } \\
\text { survival }\end{array}$ & $\begin{array}{c}\text { Yes } \\
\text { (hospitalization), } \\
\text { no (survival) }\end{array}$ & 43 \\
\hline $\begin{array}{l}\text { Dulaglutide } \\
\text { (Trulicity) } \\
2014\end{array}$ & Eli Lilly & $\begin{array}{c}\text { Type } 2 \text { diabetes } \\
\text { mellitus }\end{array}$ & Yes/Yes & 2016 & 2 & $\begin{array}{c}\text { Harvard } \\
\text { Pilgrim }\end{array}$ & $\begin{array}{l}\text { In exchange for preferred } \\
\text { status for dulaglutide } \\
\text { on Harvard Pilgrim's } \\
\text { formulary, Harvard } \\
\text { Pilgrim will pay Eli } \\
\text { Lilly a lower net price } \\
\text { for dulaglutide if fewer } \\
\text { patients taking it achieve } \\
\text { an HbAlc value of less } \\
\text { than } 8 \% \text { compared with } \\
\text { patients taking other } \\
\text { GLP-1 receptor agonists, } \\
\text { and a higher net price if } \\
\text { patients taking dulaglutide } \\
\text { have better outcomes } \\
\text { than patients taking other } \\
\text { competing drugs. }\end{array}$ & Blood sugar & No & $\begin{array}{l}44 \\
45 \\
58\end{array}$ \\
\hline $\begin{array}{l}\text { Sitagliptin } \\
\text { (Januvia) } \\
2006\end{array}$ & & & & & 10 & & $\begin{array}{l}\text { Merck will pay rebates if } \\
\text { certain portions of patients } \\
\text { in the insurer's population }\end{array}$ & & & \\
\hline $\begin{array}{l}\text { Sitagliptin } \\
\text { and } \\
\text { metformin } \\
\text { (Janumet) } \\
2007\end{array}$ & Merck & $\begin{array}{c}\text { Type } 2 \text { diabetes } \\
\text { mellitus }\end{array}$ & Yes/Yes & 2016 & 9 & Aetna & $\begin{array}{l}\text { who are prescribed the } \\
\text { drugs do not achieve treat- } \\
\text { ment goals and require } \\
\text { further therapy, as deter- } \\
\text { mined by their physicians. }\end{array}$ & $\begin{array}{l}\text { Further } \\
\text { therapy }\end{array}$ & Yes & $\begin{array}{l}40 \\
47 \\
58\end{array}$ \\
\hline $\begin{array}{l}\text { Sacubitril } \\
\text { and } \\
\text { valsartan } \\
\text { (Entresto) } \\
2015\end{array}$ & Novartis & $\begin{array}{l}\text { Chronic heart } \\
\text { failure }\end{array}$ & No/Yes & 2016 & 1 & $\begin{array}{c}\text { Harvard } \\
\text { Pilgrim }\end{array}$ & $\begin{array}{l}\text { Novartis will provide } \\
\text { Harvard Pilgrim with } \\
\text { a discount if sacubitril- } \\
\text { valsartan does not reduce } \\
\text { hospitalizations for } \\
\text { congestive heart failure by } \\
\text { a given level. }\end{array}$ & Hospitalization & Yes & $\begin{array}{l}48 \\
58\end{array}$ \\
\hline $\begin{array}{l}\text { Gefitinib } \\
\text { (Iressa) } \\
2015\end{array}$ & AstraZeneca & Lung cancer & Yes/Yes & 2015 & 0 & $\begin{array}{c}\text { Express Scripts } \\
\text { (PBM) }\end{array}$ & $\begin{array}{l}\text { AstraZeneca will reim- } \\
\text { burse costs of gefitinib if } \\
\text { a patient stops treatment } \\
\text { before third prescription } \\
\text { fill. }\end{array}$ & $\begin{array}{l}\text { Prescription } \\
\text { fill }\end{array}$ & Yes & $\begin{array}{l}49 \\
58\end{array}$ \\
\hline $\begin{array}{l}\text { Prasugrel } \\
\text { (Effient) } \\
2009\end{array}$ & Eli Lilly & $\begin{array}{c}\text { Acute coronary } \\
\text { syndrome }\end{array}$ & Yes/Yes & 2016 & 7 & Humana & $\begin{array}{l}\text { Eli Lilly will tie } \\
\text { reimbursements for } \\
\text { prasugrel to the rate of } \\
\text { hospitalizations among } \\
\text { patients who take it. }\end{array}$ & Hospitalization & Yes & $\begin{array}{l}49 \\
58\end{array}$ \\
\hline
\end{tabular}




\section{TABLE 1 Summary of Literature Review (continued)}

\begin{tabular}{|c|c|c|c|c|c|c|c|c|c|c|}
\hline 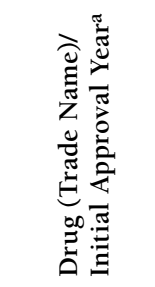 & 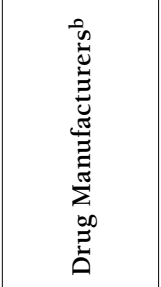 & 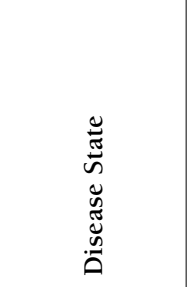 & 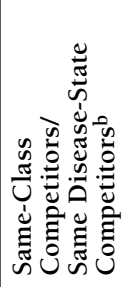 & 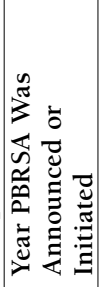 & 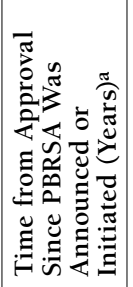 & 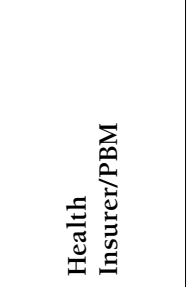 & 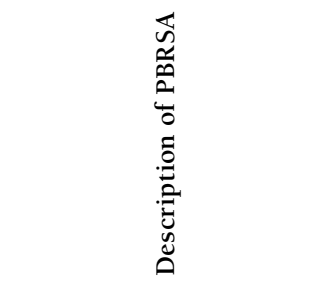 & 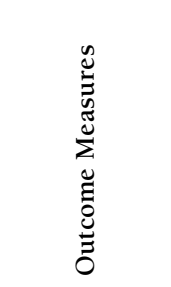 & 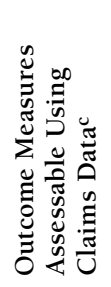 & 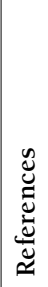 \\
\hline $\begin{array}{l}\text { Ticagrelor } \\
\text { (Brilinta) } \\
2011\end{array}$ & AstraZeneca & $\begin{array}{c}\text { Acute coronary } \\
\text { syndrome }\end{array}$ & Yes/Yes & 2016 & 5 & $\begin{array}{c}\text { University of } \\
\text { Pittsburgh } \\
\text { Medical } \\
\text { Center's } \\
\text { regional health } \\
\text { plan }\end{array}$ & $\begin{array}{l}\text { AstraZeneca will cover } \\
\text { a portion of the costs of } \\
\text { treating patients who have } \\
\text { additional heart attacks } \\
\text { after taking ticagrelor if } \\
\text { the rate of heart attacks } \\
\text { exceeds a threshold. }\end{array}$ & Heart attack & Yes & $\begin{array}{l}49, \\
58\end{array}$ \\
\hline $\begin{array}{l}\text { Teriparatide } \\
\text { (Forteo) } \\
2002\end{array}$ & Eli Lilly & Osteoporosis & No/Yes & 2017 & 15 & $\begin{array}{l}\text { Harvard } \\
\text { Pilgrim }\end{array}$ & $\begin{array}{l}\text { Eli Lilly will reduce the } \\
\text { cost of teriparatide for } \\
\text { Harvard Pilgrim if mean- } \\
\text { ingful improvements in } \\
\text { the drug's persistence are } \\
\text { achieved compared with } \\
\text { the baseline level of adher- } \\
\text { ence observed in Harvard } \\
\text { Pilgrim's population. }\end{array}$ & Persistence & Yes & $\begin{array}{l}54 \\
58\end{array}$ \\
\hline $\begin{array}{l}\text { Etanercept } \\
\text { (Enbrel) } \\
1998\end{array}$ & Amgen & $\begin{array}{l}\text { Rheumatoid } \\
\text { arthritis }\end{array}$ & Yes/Yes & 2017 & 19 & $\begin{array}{l}\text { Harvard } \\
\text { Pilgrim }\end{array}$ & $\begin{array}{l}\text { The 2-year contract for } \\
\text { etanercept is based on an } \\
\text { effectiveness algorithm } \\
\text { consisting of } 6 \text { criteria } \\
\text { (including patient compli- } \\
\text { ance, switching or adding } \\
\text { drugs, dose escalation, } \\
\text { and steroid interventions) } \\
\text { that serve as a global mea- } \\
\text { sure of the drug's impact. } \\
\text { Harvard Pilgrim will } \\
\text { pay less for etanercept if } \\
\text { patient scores are below } \\
\text { a specified level. The } \\
\text { contract also specifies the } \\
\text { tracking of patient adher- } \\
\text { ence to the drug. }\end{array}$ & $\begin{array}{l}\text { Compliance/ } \\
\text { adherence, } \\
\text { switching } \\
\text { drugs, adding } \\
\text { drugs, dose } \\
\text { escalation, } \\
\text { steroid } \\
\text { interventions } \\
\text { (Last criteria } \\
\text { not stated) }\end{array}$ & Yes & $\begin{array}{l}55 \\
58\end{array}$ \\
\hline
\end{tabular}

a Based on the first indication approved by the U.S. Food and Drug Administration.

${ }^{b}$ At the time the PBRSA was announced or initiated.

cBased on the assumption that claims data would be an appropriate data source; electronic medical record data were assumed to be an appropriate source for all other outcomes.

$C E D=$ coverage with evidence determination; $C M S=$ Centers for Medicare $\&$ Medicaid Services; GLP-1 = glucagon-like peptide-1; HbAlc=hemoglobin Alc; Hct =hematocrit; $L D L=$ low-density lipoprotein; $M P R=$ medication possession ratio; $N / A=$ not applicable (drug not approved at the time for treatment of the corresponding disease state and thus had no actual competitors); $P B M=$ pharmacy benefit manager; PBRSA= performance-based risk-sharing arrangement; SVR=sustained virologic response.

multiple sources presented the same information on a PBRSA, the earliest-published source was included in Table 1, which summarizes the results; other sources were also included in Table 1 if they provided additional information on a PBRSA beyond that of the earliest-published source.

In total, 26 PBRSAs were identified. Among them, 16 (62\%) were announced or initiated from 2015 to 2017, and 10 (38\%) were announced or initiated from 1997 to 2012. In relation to disease state, 13 (50\%) PBRSAs involved cardiometabolic indications (i.e., type 2 diabetes mellitus, acute coronary syn- drome, hyperlipidemia, and chronic heart failure); 5 (19\%) involved oncology indications (i.e., colorectal cancer and lung cancer); and 8 (31\%) involved other indications (i.e., anemia, osteoporosis, multiple sclerosis, hepatitis $C$, and rheumatoid arthritis; Table 1).

Categorized by health insurer or PBM, 10 (38\%) PBRSAs involved large multistate insurers (Cigna, $\mathrm{n}=6$; Humana, $\mathrm{n}=2$; and Aetna, $n=2) ; 5$ (19\%) involved the Centers for Medicare \& Medicaid Services; 7 (27\%) involved regional insurers (Harvard Pilgrim, $\mathrm{n}=5$; Health Alliance, $\mathrm{n}=1$; and University 
of Pittsburgh Medical Center's regional health plan, $\mathrm{n}=1$ ); 3 (12\%) involved PBMs (Prime Therapeutics, CVS Health, and Express Scripts); and 1 (4\%) involved multiple unspecified insurers. The drug manufacturer most actively involved with initiating PBRSAs was Amgen ( $\mathrm{n}=5,19 \%)$, followed by Novartis ( $n=4,15 \%)$, Sanofi $(n=3,12 \%)$, Merck $(n=3,12 \%)$, and Eli Lilly ( $\mathrm{n}=3,12 \%$; Table 1 ).

As for FDA approval date for a treatment, 15 (58\%) PBRSAs were announced or initiated within 5 years after approval, and $11(42 \%)$ were announced or initiated more than 5 years after approval. For PBRSAs in which the drug had a same-class competitor and/or same disease-state competitor at the time, $17(65 \%)$ had a same-class competitor and same disease-state competitor; 5 (19\%) had only a same disease-state competitor (i.e., drugs had novel mechanisms of action); and 4 (15\%) were unique in that reimbursement was based on coverage with evidence development (CED), meaning that the drugs were not yet approved for the treatment of the corresponding disease states and thus had no actual competitors (Table 1).

For the data collection method, EMR data would have been an appropriate source for 12 (46\%) PBRSAs; claims data would have been an appropriate source for 11 (42\%) PBRSAs; and EMR and claims data would have been appropriate sources for 2 (8\%) PBRSAs. An appropriate data source could not be determined for 1 (4\%) PBRSA because a description of its outcome measures was not available.

Outcomes that could be measured using claims data include hospitalizations $(n=5)$, adherence/compliance $(n=5)$, persistence $(n=1)$, switching drugs $(n=1)$, adding drugs $(n=1)$, dose escalation ( $n=1)$, steroid interventions $(n=1)$, emergency room visits $(n=1)$, nonspinal fractures $(n=1)$, cost $(n=1)$, further therapy $(n=1)$, prescription fills $(n=1)$, and heart attacks $(n=1)$. Outcomes that could be measured using EMR data include low-density lipoprotein $(n=5)$, survival $(n=5)$, blood sugar $(n=2)$, hematocrit $(n=1)$, and sustained virologic response $(\mathrm{n}=1$; Table 1$)$.

\section{Discussion}

This systematic review identified 26 U.S.-specific PBRSAs for drugs that were announced or initiated from 1997 to 2017. More than half occurred from 2015 to 2017, suggesting a change from what appeared to be a slowing pace of initiation of PBRSAs worldwide in 2012 and 2013..$^{9}$ Furthermore, these U.S.-specific findings are unique in that only $15 \%$ of the identified PBRSAs involved CED, and only 19\% involved oncology indications. These findings compare with $41 \%$ and $52 \%$ of PBRSAs worldwide (92\% which were for drugs) that involved CED and oncology indications, respectively. ${ }^{9}$ These observed differences between the United States and other countries reflect the priorities of regional stakeholders and the extent to which they publicly disclosed PBRSAs.
At a basic level, PBRSAs create access to drugs for patients, while addressing uncertainty in clinical and economic outcomes, and can thus function as effective coverage and reimbursement mechanisms. ${ }^{9}$ Drug manufacturers benefit from PBRSAs by ensuring coverage for specific drugs, while health insurers and PBMs benefit by linking a drug's price to its realworld performance or clinical efficacy (in the case of CED). With drug pricing under increased scrutiny from the public, health insurers, and PBMs, in conjunction with the recent launch of high-cost specialty agents and the aggressive pricing practices of several generic drug manufacturers, ${ }^{56}$ it is likely that this upward trend in PBRSAs will continue.

\section{Factors Associated with PBRSAs}

Various factors are associated with the implementation of the PBRSAs identified in this review. Examples of such factors include the recent launch of a high-grossing treatment (i.e., sofosbuvir), ${ }^{57}$ same-class competitors, and same disease-state competitors. With the 2013 launch of sofosbuvir, one of the best-selling drugs in its first year on the market, ${ }^{57}$ health insurers likely became much more sensitized to the budget impacts associated with the inclusion of costly drugs on their formularies, which may have influenced the uptick in PBRSAs in 2015 and 2016.

Also, among drugs in which PBRSAs were identified, most had competitors (within the same class, for the same disease state, or both) at the time of initiation of the PBRSAs..$^{58}$ Competitive pressure creates competition for drug access to patients, especially for costly agents; for example, the initiation of a PBRSA for ledipasvir-sofosbuvir in $2015,{ }^{40} 1$ year after its approval and the introduction of its competitor ombitasvirparitaprevir-ritonavir. ${ }^{58}$ Furthermore, many drugs for which we identified PBRSAs appear to lack head-to-head clinical trials with the standard of care for their approved indications. ${ }^{58}$ At the time of approval for these drugs, and before the PBRSAs were initiated, there was likely considerable uncertainty with regards to their efficacy and safety versus the standard of care. Combined with a lack of real-world evidence at the time of drug approval, the previously mentioned factors represent compelling reasons for health insurers and PBMs to engage with drug manufacturers to initiate PBRSAs.

The high per-member-per-year (PMPY) spend on various therapeutic areas contributes to the needs of health insurers for more value-based approaches to cost management. In the context of Express Scripts's 2016 spend for its commercially insured population and the PBRSAs that were identified in this systematic review, diabetes ( $\$ 108.80$ PMPY), hyperlipidemia (\$38.45 PMPY), high blood pressure/heart disease ( $\$ 34.52$ PMPY), multiple sclerosis (MS; \$58.63 PMPY), oncology (\$60.70 PMPY), and hepatitis C (\$25.26 PMPY) ranked in the top 15 therapy classes. ${ }^{59}$ Among traditional drugs, sitagliptin ranked sixth in PMPY spend in 2016 (\$8.66 PMPY). ${ }^{59}$ 
Based on the assumption of similar trends for other health insurers in diabetes drug spending in previous years and the availability of other approved dipeptidyl peptidase-4 inhibitors, this assumption may explain Merck's willingness to engage in a PBRSA for sitagliptin and sitagliptin-metformin with Aetna in 2016. ${ }^{46,47}$ Furthermore, among specialty drugs, ledipasvirsofosbuvir ranked fifth in PMPY spend in 2016 (\$9.86); fingolimod ranked seventh in PMPY spend in 2016 (\$8.48); and among MS drugs, interferon beta-la ranked fourth in PMPY spend in 2016. ${ }^{59}$ Assuming similar spending trends for other health insurers in previous years, and given the availability of other competitors for the 3 previously mentioned specialty drugs, PBRSAs were likely initiated for each of them in order to manage drug costs.

Drug spending appears to be associated with the initiation of specific PBRSAs, which will most likely continue trending upwards as health insurers and PBMs attempt to separate drug spending from prescription volume. In addition, a lack of transparency among all stakeholders involved in PBRSAs will likely persist into the future. ${ }^{9}$ Unless stakeholders publicly disclose PBRSAs, their relation to costly therapy classes, costly drug classes, and other factors will be unknown.

Over half of the PBRSAs identified in this systematic review involved drugs with cardiometabolic indications. Given the large number of Americans affected by diabetes (30.3 million) and coronary heart disease (15.4 million, inclusive of myocardial infarction, angina pectoris, heart failure, stroke, and congenital cardiovascular defects), ${ }^{60,61}$ it is not surprising that health insurers and PBMs are interested in managing the high costs associated with these disease states. ${ }^{59}$ As the American population continues to age and the proportion of individuals aged 65 years and above continues to grow, health insurers and PBMs will increasingly focus on managing costs of drugs with the potential for high budget impact. PBRSAs can offer an attractive alternative to traditional rebates for high-cost drugs or those with large target populations. Health insurers can align payments for drugs with the value they create, while drug manufacturers in turn will receive incentives to continue developing new treatments.

\section{Implementation of PBRSAs}

To establish viable PBRSAs, stakeholders should evaluate key factors such as the availability of existing data systems, existence of mutually acceptable measures (ideally with shortterm responses), and reasonable proxies for clinical outcomes (if needed). ${ }^{9}$ Low administrative burden and acceptable monitoring and evaluation costs are also critical to the successful implementation of PBRSAs. ${ }^{8}$ Based on the outcome measures, multiple PBRSAs with certain health insurers, and data collection methods that were identified in this systematic review, it appears that many of the previously mentioned barriers to implementation have been overcome by stakeholders. Given the availability of claims data, ${ }^{8}$ the frequent use of claims data by PBMs and health insurers,,$^{23}$ and the frequent use of real-world evidence by manufacturers, ${ }^{23}$ the following discussion will focus on outcome measures available in claims data to better understand the considerations and implications of their use in PBRSAs.

The number of prescription fills for a drug and persistence on a drug (i.e., whether a patient's length of therapy meets or exceeds a specified threshold ${ }^{62}$ ) represent measures contained in claims data that can serve as proxies for drug effectiveness. These measures are based on the assumption that patients who continue taking specific drugs do so because the drugs are working for them. Claims data also allow for an evaluation of adherence (also referred to as compliance ${ }^{62}$ ). Although it is not explicitly stated for most of the PBRSAs identified in this systematic review, adherence provisions (e.g., using medication possession ratio) likely exist for many arrangements where outcomes can be influenced by whether a patient reliably takes a drug ${ }^{26}$; this is observed in the cases of evolocumab, ${ }^{26}$ interferon beta- $1 a,{ }^{36-38}$ and etanercept. ${ }^{55}$ Despite the utility of measuring adherence in PBRSAs, interpretations of adherence will always be subject to the limitation of unknown timing of doses and whether patients actually took their doses. ${ }^{62}$

Other measures contained in claims data that can serve as proxies for actual outcomes include hospitalizations, emergency room visits, further therapy, switching drugs, adding drugs, dose escalation, and steroid interventions. These measures estimate disease worsening in the absence of claims data for the clinical stage of a disease. In the case of hospitalizations and emergency room visits, these outcomes also have direct cost implications that are of interest to health insurers. In the context of costs, however, no PBRSA identified in this review evaluated the performance of a drug based on costs before and after treatment initiation. Such an approach would directly address concerns of health insurers regarding the budgetary impact of treatments but would not incorporate other important aspects of value such as comparative effectiveness, comparative safety, cost-effectiveness, mechanism of action, indirect costs, and mode of administration.

\section{Limitations}

This systematic review has some limitations to consider. The main limitation is the lack of publicly available data on every PBRSA between drug manufacturers and health insurers or PBMs. Taking into account the PBRSAs identified in this review, it should be noted that they likely represent a minority of the total number in place between drug manufacturers and health insurers or PBMs. Anthem, for example, does not publicly disclose its PBRSAs, along with many drug manufacturers. ${ }^{63}$ Humana had 15 PBRSAs for drugs in effect as of November 2016, but only 2 were identified in this review; UnitedHealth Group had 1 in effect as of May 2016 but did not provide 
details; and Aetna only confirmed 2 as of November 2016 but would not disclose possible others. ${ }^{63}$ This lack of public disclosure is further reinforced by a search of the 2015 annual reports of pharmaceutical companies, health insurance companies, and PBMs (see Methods section for details), which yielded no specific data on PBRSAs and thus supported previous findings that many are not publicly disclosed. ${ }^{9}$

\section{Conclusions}

The number of publicly disclosed U.S.-specific PBRSAs for drugs has increased over the years as the need to demonstrate value for money has become more important in paying for health care. The findings of this systematic review likely underestimate the total number of PBRSAs but, nonetheless, confirm the interest of stakeholders in such arrangements and their confidence in the use of the selected outcome measures. Also, our results suggest that barriers to initiating PBRSAs have decreased over time. Finally, each PBRSA represents a timely collaboration among stakeholders to provide access to a drug, while generating evidence to better elucidate its clinical and economic value.

\section{Authors}

JUSTIN S. YU, PharmD, MS, Allergan, Irvine, California. LAUREN CHIN, PharmD, and JENNIFER OH, BS, University of Washington, School of Pharmacy, Seattle. JORGE FARIAS, PharmD, MS, BluePath Solutions, Los Angeles, California.

AUTHOR CORRESPONDENCE: Justin S. Yu, PharmD, MS, Manager, Global Health Economics and Outcomes Research, Allergan, 2525 Dupont Dr., Irvine, CA 92612. Tel.: 626.824.7896; E-mail: justinyu@uw.edu.

\section{DISCLOSURES}

No funding supported this systematic review. Yu is an employee and shareholder of Allergan. Chin reports personal fees from Formulary Resources. Oh and Farias have nothing to disclose.

Study concept and design were primarily contributed by $\mathrm{Yu}$, along with the other authors. All authors contributed to the collection and interpretation of the data. The manuscript was written by Yu, Chin, Oh, and Farias and revised by $\mathrm{Yu}$ and $\mathrm{Chin}$, along with the other authors.

\section{REFERENCES}

1. Porter ME. What is value in health care? N Engl J Med. 2010;363(26):2477-81.

2. Miller DW. Value-based pricing: examples of healthcare system reforms from the UK and U.S. and implications for industry. Pharmaceut Med. 2012;26(4):217-22

3. Antoñanzas F, Terkola R, Postma M. The value of medicines: a crucial but vague concept. Pharmacoeconomics. 2016;34(12):1227-39.

4. Amgen. Chairman and CEO letter and Amgen Inc. 2015 annual report. March 31, 2016. Available at: http://investors.amgen.com/phoenix. zhtml?c=61656\&p=irol-reportsannual. Accessed August 5, 2017.
5. Sanofi. Form 20-F. 2015. Available at: http://en.sanofi.com/ Images/45889_Sanofi_20-F_2015_V2.pdf. Accessed August 5, 2017.

6. QuintilesIMS. IMS health study: U.S. drug spending growth reaches 8.5 percent in 2015. April 14, 2016. Available at: https://www.imshealth.com/ en/about-us/news/ims-health-study-us-drug-spending-growth-reaches-8.5percent-in-2015. Accessed August 5, 2017.

7. Sorenson C. Use of comparative effectiveness research in drug coverage and pricing decisions: a six-country comparison. Issue Brief (Commonw Fund). 2010;91:1-14. Available at: http://www.commonwealthfund.org/ /media/ Files/Publications/Issue\%20Brief/2010/Jul/1420_Sorenson_Comp_Effect_ intl_ib_71.pdf. Accessed August 5, 2017.

8. Garrison LP Jr, Towse A, Briggs A, et al. Performance-based risk-sharing arrangements-good practices for design, implementation, and evaluation: report of the ISPOR Good Practices for Performance-Based Risk-Sharing Arrangements Task Force. Value Health. 2013;16(5):703-19.

9. Carlson JJ, Gries KS, Yeung K, Sullivan SD, Garrison LP Jr. Current status and trends in performance-based risk-sharing arrangements between healthcare payers and medical product manufacturers. Appl Health Econ Health Policy. 2014;12(3):231-38.

10. Carlson JJ, Sullivan SD, Garrison LP, Neumann P, Veenstra DL. Linking payment to health outcomes: a taxonomy and examination of performance-based reimbursement schemes between healthcare payers and manufacturers. Health Policy. 2010;96(3):179-90.

11. Daniel GW, Rubens EK, McClellan M. Coverage with evidence development for Medicare beneficiaries: challenges and next steps. JAMA Intern Med. 2013;173(14):1281-82.

12. Schnipper LE, Davison NE, Wollins DS, et al. Updating the American Society of Clinical Oncology value framework: revisions and reflections in response to comments received. J Clin Oncol. 2016;34(24):2925-34.

13. National Comprehensive Cancer Network. NCCN Evidence Blocks. Frequently asked questions. 2016. Available at: https://www.nccn.org/ evidenceblocks/pdf/EvidenceBlocksFAQ.pdf. Accessed August 5, 2017.

14. Institute for Clinical and Economic Review. Recent reports. 2016. Available at: https://icer-review.org/materials/. Accessed August 5, 2017.

15. Memorial Sloan Kettering Cancer Center. Drug Pricing Lab. DrugAbacus Tools. 2016. Available at: http://www.drugabacus.org/drug-abacus/tool/. Accessed August 5, 2017

16. Express Scripts. 2015 drug trend report. March 2016. Available at: https://lab.express-scripts.com/lab/ /media/d82d33d9686a4c5aab3labb87ecdc73a.ashx. Accessed August 21, 2017.

17. CVS Health. Specialty costs: can they be contained? 2016. Available at: https://cvshealth.com/thought-leadership/cvs-health-research-institute/ specialty-costs-can-they-be-contained. Accessed August 5, 2017.

18. Lotvin AM, Shrank WH, Singh SC, Falit BP, Brennan TA. Specialty medications: traditional and novel tools can address rising spending on these costly drugs. Health Aff (Millwood). 2014;33(10):1736-44.

19. Centers for Medicare \& Medicaid Services. National health expenditure projections 2015-2025. Available at: https://www.cms.gov/Research-StatisticsData-and-Systems/Statistics-Trends-and-Reports/NationalHealthExpendData/ Downloads/proj2015.pdf. Accessed August 5, 2017.

20. Ernst \& Young. Strong growth for major pharmaceutical companies in 2015-big biotech is driving growth and putting big pharma under pressure. News release. 2016. Available at: http://www.ey.com/Publication/ vwLUAssets/EY_news_release_Pharma_Bilanzanalyse_2016/\$FILE/ EY-news-release-Pharma-Bilanzanalyse-2016.pdf. Accessed August 5, 2017.

21. Forbes. America's biggest health insurance providers. 2015. Available at: https://www.forbes.com/pictures/geji45fhhm/l-unitedhealth-group/ \#558c0553115d. Accessed August 5, 2017. 
22. Balto DA. Testimony of David A. Balto. "The state of competition in the pharmacy benefits manager and pharmacy marketplaces." House Judiciary Subcommittee on Regulatory Reform, Commercial and Antitrust Law. November 17, 2015. Available at: https://judiciary.house.gov/wp-content/ uploads/2016/02/Balto-Testimony-1.pdf. Accessed August 5, 2017.

23. IMS Health. Why pharma needs to work differently with payers and IDNs on RWE. White paper. May 2015. Available at: https://www.imshealth. com/files/web/Global/Services/Services\%20TL/why_pharma_rwe_white_ paper.pdf. Accessed August 5, 2017.

24. Carino T, Williams RD II, Colbert AM, Bridger P. Medicare's coverage of colorectal cancer drugs: a case study in evidence development and policy. Health Aff (Millwood). 2006;25(5):1231-39.

25. Moldrup C. No cure, no pay. BMJ. 2005;330(7502):1262-64.

26. Barlas $\mathrm{S}$. Health plans and drug companies dip their toes into valuebased pricing: the pressure is on P\&T committees to monitor utilization. P T. 2016:41(1):39-53.

27. Berns JS, Fishbane S, Elzein H, et al. The effect of a change in epoetin alfa reimbursement policy on anemia outcomes in hemodialysis patients. Hemodialy Int. 2005;9(3):255-63.

28. Carlson JJ, Garrison LP Jr, Sullivan SD. Paying for outcomes: innovative coverage and reimbursement schemes for pharmaceuticals. J Manag Care Pharm. 2009;15(8):683-87. Available at: http://www.jmcp.org/doi/10.18553/ jmcp.2009.15.8.683.

29. Dalzell MD. An old-fashioned notion: stand behind your wares. Manag Care. 2017;26(1):8-9.

30. Towse A, Garrison LP Jr. Can't get no satisfaction? Will pay for performance help? Toward an economic framework for understanding performance-based risk-sharing agreements for innovative medical products. Pharmacoeconomics. 2010;28(2):93-102.

31. Neuman PJ, Chambers JD, Simon F, Meckley LM. Risk-sharing arrangements that link payment for drugs to health outcomes are proving hard to implement. Health Aff (Millwood). 2011;30(12):2329-37.

32. Carroll E, Truglio A. A risk-benefit analysis of key performance-based risk sharing agreements (PBRSAs) in the EU and U.S. (2002-2016). Value Health. 2016;19(7):A500 [Abstract PHP342]. Available at: http://www. valueinhealthjournal.com/article/S1098-3015(16)32257-4/abstract. Accessed August 5, 2017.

33. Ferrario A, Nicod E, Kanavos P. Evidence on the impact of managed entries on payers, patients, manufacturers, and health care workers. Value Health. 2011;14(7):A360-A361 [Abstract PHP151]. Available at: http://www. valueinhealthjournal.com/article/S1098-3015(11)02263-7/abstract. Accessed August 5, 2017.

34. Cigna. CIGNA and Merck sign performance-based contract. Business Wire. April 23, 2009. Available at: http://www.businesswire.com/news/ home/20090423005503/en/CIGNA-Merck-Sign-Performance-BasedContract. Accessed August 5, 2017.

35. PR Newswire. Health alliance announces first fracture protection program for Actonel (risedronate sodium) tablets. April 14, 2009. Available at: http://www.prnewswire.com/news-releases/health-alliance-announcesfirst-fracture-protection-program-for-actonelr-risedronate-sodiumtablets-61811307.html. Accessed August 5, 2017.

36. Cigna. Cigna and EMD Serono team to fight multiple sclerosis under first outcomes-based specialty medication contract. News release. March 16, 2011. Available at: http://www.cigna.com/newsroom/news-releases/2011/ pdf/cigna-and-emd-serono-team-to-fight-multiple-sclerosis-under-firstoutcomes-based-specialty-medication-contract.pdf. Accessed August 5, 2017.

37. AISHealth. Cigna signs outcomes-based contract that may be first deal for specialty drug. April 2011. Available at: https://aishealth.com/archive/ nspn0411-01. Accessed August 5, 2017.
38. PR Newswire. Prime Therapeutics announces CareCentered Contract with EMD Serono, Inc. for MS drug Rebif. March 7, 2012. Available at: http://www.prnewswire.com/news-releases/prime-therapeutics-announcescarecentered-contract-with-emd-serono-inc-for-ms-drug-rebif-141779863. html. Accessed August 5, 2017.

39. Roland D. Novartis looks at ways to win over health insurers. MarketWatch. July 10, 2015. Available at: http://www.marketwatch.com/ story/novartis-looks-at-ways-to-win-over-health-insurers-2015-07-10. Accessed August 5, 2017.

40. Cigna. Cigna signs agreement with Gilead to improve affordability of hepatitis $C$ treatment for customers and clients. News release. February 4 , 2015. Available at: http://www.cigna.com/newsroom/news-releases/2015/ cigna-signs-agreement-with-gilead-to-improve-affordability-of-hepatitis-ctreatment-for-customers-and-clients. Accessed August 5, 2017.

41. Cigna. Cigna's two new value-based contracts with pharma for PCSK9 inhibitor cholesterol drugs tie financial terms to improved customer health. News release. May 11, 2016. Available at: http://www.cigna.com/ newsroom/news-releases/2016/pdf/cignas-two-new-value-based-contractswith-pharma-for-pcsk9-inhibitor-cholesterol-drugs-tie-financial-terms-toimproved-customer-health.pdf. Accessed August 5, 2017.

42. Cigna. Cigna implements value-based contract with Novartis for heart drug Enresto. News release. February 8, 2016. Available at: http://www. cigna.com/newsroom/news-releases/2016/cigna-implements-value-basedcontract-with-novartis-for-heart-drug-entrestotm. Accessed August 5, 2017.

43. Humer C. Novartis sets heart-drug price with two insurers based on health outcome. Reuters. February 9, 2016. Available at: http://www.reuters. com/article/us-cigna-novartis-drugpricing-idUSKCNOVH25K. Accessed August 5, 2017.

44. Weisman R. Harvard Pilgrim adds new drugs under "pay-forperformance" deals. Boston Globe. June 27, 2016. Available at: https://www. bostonglobe.com/business/2016/06/27/harvard-pilgrim-adds-new-drugsunder-pay-for-performance-deals/2OQYId32R5RSc0s8wGKooM/story.html. Accessed August 5, 2017.

45. Harvard Pilgrim Health Care. Harvard Pilgrim signs value-based contract with Eli Lilly for Trulicity. News release. June 27, 2016. Available at: https://www.harvardpilgrim.org/pls/portal/url/ITEM/9FDFE06CBD2B43E79 8954E60EE6F70D6. Accessed August 5, 2017.

46. Aetna. Aetna and Merck sign a unique value-based contract for Januvia and Janumet. Business Wire. October 11, 2016. Available at: https://news. aetna.com/news-releases/aetna-and-merck-sign-a-unique-value-basedcontract-for-januvia-and-janumet/. Accessed August 5, 2017.

47. George J. Aetna, Merck sign value-based pact for diabetes drugs. Philadelphia Business Journal. October 11, 2016. Available at: http://www. bizjournals.com/philadelphia/news/2016/10/11/aetna-merck-sign-valuebased-pact-diabetes-januvia.html. Accessed August 5, 2017.

48. Harvard Pilgrim Health Care. Harvard Pilgrim Health Care is first regional health plan to execute pay-for-performance deal with Novartis for Entresto. News release. June 2016. Available at: https://www.harvardpilgrim. org/pls/portal/url/ITEM/ACAE90F9833C4AF9B5E088E0C67FE96B. Accessed August 5, 2017.

49. Loftus P, Mathews AW. Health insurers push to tie drug prices to outcomes. The Wall Street Journal. May 11, 2016. Available at: http:// www.wsj.com/articles/health-insurers-push-to-tie-drug-prices-tooutcomes-1462939262. Accessed August 5, 2017.

50. Amgen. Amgen announces Repatha preferred position on CVS Health commercial formularies. News release. November 23, 2015. Available at: http://www.amgen.com/media/news-releases/2015/11/amgen-announcesrepatha-preferred-position-on-cvs-health-commercial-formularies/. Accessed August 5, 2017.

51. Harvard Pilgrim Health Care. PCSK9 inhibitors: prior authorizations and innovative contract for Repatha. December 2015. Available at: https://www.harvardpilgrim.org/portal/page?_pageid=253,8324049\&_ $\mathrm{dad}=$ portal\&_schema=PORTAL. Accessed August 5, 2017. 
52. Centers for Medicare \& Medicaid Services. Off-label use of colorectal cancer drugs. March 19, 2015. Available at: https://www.cms.gov/Medicare/ Coverage/Coverage-with-Evidence-Development/Off-label-use-of-ColorectalCancer-Drugs.html. Accessed August 5, 2017.

53. Centers for Medicare \& Medicaid Services. NCI/CTEP-sponsored studies selected for inclusion in NCI-CMS pilot project. March 8, 2006. Available at: https://www.cms.gov/Medicare/Coverage/DeterminationProcess/downloads/ id90b.pdf. Accessed August 5, 2017.

54. Harvard Pilgrim Health Care. Harvard Pilgrim signs value-based contract with Eli Lilly \& Company for its osteoporosis drug, Forteo. February 22, 2017. Available at: https://www.harvardpilgrim.org/public/news-detail? $\mathrm{nt}=\mathrm{HPH}$ _News_C\&nid=1471912468207. Accessed August 5, 2017.

55. Harvard Pilgrim Health Care. Harvard Pilgrim signs outcomesbased contract with Amgen for Enbrel. February 22, 2017. Available at: https://www.harvardpilgrim.org/public/news-detail?nt=HPH News_C\&nid=1471912468296. Accessed August 5, 2017.

56. Mangan D. Drug price shock: Feds reveal how medication costs hit Medicare and Medicaid. CNBC. November 14, 2016. Available at: https:// finance.yahoo.com/news/drug-price-shock-feds-reveal-194026080.html. Accessed August 5, 2017.

57. Polack A. Sales of Sovaldi, new Gilead hepatitis C drug, soar to $\$ 10.3$ billion. The New York Times. February 3, 2015. Available at: http://www. nytimes.com/2015/02/04/business/sales-of-sovaldi-new-gilead-hepatitis-cdrug-soar-to-10-3-billion.html?_r=0. Accessed August 5, 2017.
58. U.S. Food \& Drug Administration. Drugs@FDA: FDA approved drug products. 2016. Available at: http://www.accessdata.fda.gov/scripts/cder/daf/. Accessed August 5, 2017.

59. Express Scripts. 2016 commercial drug trend report. 2017. Available at: https://lab.express-scripts.com/lab/drug-trend-report/commercial-drugtrend-report. Accessed August 5, 2017.

60. National Center for Chronic Disease Prevention and Health Promotion. National diabetes statistics report, 2017. Available at: https://www.cdc.gov/ diabetes/pdfs/data/statistics/national-diabetes-statistics-report.pdf. August 21, 2017.

61. Go AS, Mozaffarian D, Roger VL, et al. Heart disease and stroke statistics-2014 update. Circulation. 2014;129(3):e28-e292. Available at: http://circ.ahajournals.org/content/circulationaha/early/2013/12/18/01. cir.0000441139.02102.80.full.pdf. Accessed August 5, 2017.

62. Fairman K, Motheral B. Evaluating medication adherence: which measure is right for your program? J Manag Care Pharm. 2000;6(6):499-504. Available at: http://www.jmcp.org/doi/pdf/10.18553/jmcp.2000.6.6.499.

63. Kelly C. US outcomes-based contracts: big uptick in interest, but not execution. In Vivo. November 6, 2016. Available at: https://invivo.pharmamedtechbi.com/IV004953/US-OutcomesBased-Contracts-Big-Uptick-InInterest-But-Not-Execution. Accessed August 5, 2017. 